\title{
Assessment of Geometric Distortion in Six Clinical Scanners Using a 3D-Printed Grid Phantom
}

\author{
Maysam Jafar, Yassir M. Jafar, Christopher Dean (iD) and Marc E. Miquel * \\ Clinical Physics, Barts Health NHS Trust, London EC1A 7BE, UK; maysam.jafar@mriphysics.net (M.J.); \\ yassir.jafar@mriphysics.net (Y.M.J.); chris.dean@bartshealth.nhs.uk (C.D.) \\ * Correspondence: marc.miquel@bartshealth.nhs.uk; Tel.: +44-203-765-8801
}

Received: 26 May 2017; Accepted: 15 July 2017; Published: 18 July 2017

\begin{abstract}
A cost-effective regularly structured three-dimensional (3D) printed grid phantom was developed to enable the quantification of machine-related magnetic resonance (MR) distortion. This phantom contains reference features, "point-like" objects, or vertices, which resulted from the intersection of mesh edges in 3D space. 3D distortions maps were computed by comparing the locations of corresponding features in both MR and computer tomography (CT) data sets using normalized cross correlation. Results are reported for six MRI scanners at both $1.5 \mathrm{~T}$ and $3.0 \mathrm{~T}$ field strengths within our institution. Mean Euclidean distance error for all MR volumes in this study, was less than $2 \mathrm{~mm}$. The maximum detected error for the six scanners ranged from $2.4 \mathrm{~mm}$ to $6.9 \mathrm{~mm}$. The conclusions in this study agree well with previous studies that indicated that MRI is quite accurate near the centre of the field but is more spatially inaccurate toward the edges of the magnetic field.
\end{abstract}

Keywords: quality analysis; MRI; distortion; 3D printing

\section{Introduction}

Major hardware-related geometric distortions in magnetic resonance imaging (MRI) arise from gradient field non-linearity and resonance offsets (encompassing chemical shifts and static field inhomogeneity) [1]. Geometric distortions occurring due to non-linearity in the gradient fields outweigh those occurring due to static field inhomogeneity as modern superconducting MRI systems are equipped with active and passive shimming technologies. For an accurate mapping of geometrical distortion in three-dimensions, two conditions must be satisfied. First, the number of control points must be sufficiently large to provide a comprehensive mapping of the spatial variation of distortion and second, the positional accuracy of these control points must be ensured [2]. Geometric distortion has been studied in two dimensions by a number of investigators using square grids [3-5], cylindrical rods [6-8], and capillary tubes [9]. A confounding limitation in two-dimensional (2D) studies is that only two coordinates define the location of the reference feature in the imaging plane and the third coordinate is undefined. For a complete mapping of geometric distortion in MRI, a three-dimensional (3D) structure is required to enable defining reference features in three dimensions. There have been few reports of mapping of geometric distortion in $3 \mathrm{D}[2,10,11]$. Breeuwer et al. described [11] a spherical phantom consisting of a 3D array of 427 spherical reference structures at positions with a manufacturing error of less than $0.05 \mathrm{~mm}$. The size of the spheres was $11 \mathrm{~mm}$ in diameter, which put a limit on the number of spheres that could be arranged in the phantom. Distortion was measured by storing the positions of the reference structures together with a point-based registration algorithm. Wang et al. [2] described a cuboid phantom consisting of $14.28 \mathrm{~mm} \times 14.39 \mathrm{~mm} \times 9.0 \mathrm{~mm}$ Perspex grid sheets typically used for building ventilation. The phantom contained 15 grid sheets, each grid containing $19 \times 19$ points on each side of the grid sheet, thus creating a total of 10,830 $(19 \times 19 \times 30)$ points that were used as reference features. The positions of reference features were determined by 
convolving a 2D cross-like template in the $x y$ plane. The authors did not mention the size of the template but stated that the size of the template is irrelevant in the analysis. Baldwin et al. [10] used a phantom similar to the one described in [2], containing $9826(2 \times 17 \times 289)$ points. The authors evaluated distortions due to inhomogeneity in the magnetic field and nonlinearity in the applied gradients by using a second reversed gradient acquisition.

In this work, we describe a novel 3D-printed grid phantom containing 891 reference features (vertices), which can be used to measure spatial distortion in three dimensions. We utilize CT images of the phantom as the ground truth data and compare the spatial locations of the reference features against each of the six MR datasets. To achieve this, we employ a 3D normalized cross correlation template matching strategy to detect the spatial distribution of the vertices. Finally, we report a combined distortion error of inhomogeneity in the magnetic field and nonlinearity in the applied gradients for six clinical MRI scanners.

\section{Materials and Methods}

\subsection{D-Printed Phantom}

A rigid $170 \mathrm{~mm} \times 170 \mathrm{~mm} \times 250 \mathrm{~mm}$ mesh cuboid insert with a $2 \mathrm{~mm}$ wireframe was designed in SketchUp (Trimble Navigation Limited, Sunnyvale, CA, USA). This rigid 3D grid was printed by a company (Shapeways BV, Eindhoven, The Netherlands) with a stated spatial error margin of $\pm 0.15 \mathrm{~mm}$ using a non-porous white plastic PA 2200 (a fine-powder on the basis of polyamide 12) and was fixated inside a $240 \mathrm{~mm} \varphi \times 250 \mathrm{~mm}$ cylinder. In Figure 1a,b, orthographic projections of the 3D model, top view, and side view, are shown respectively. A perspective view (azimuth $45^{\circ}$, elevation $20^{\circ}$ ) and a photograph of the actual phantom respectively are also shown, in Figure 1c, d respectively.
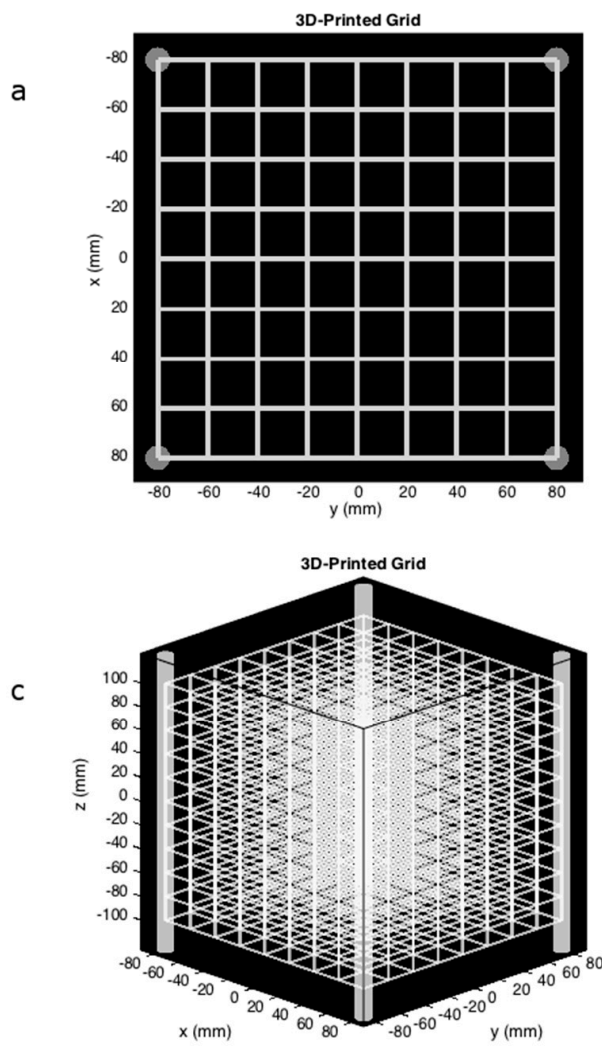
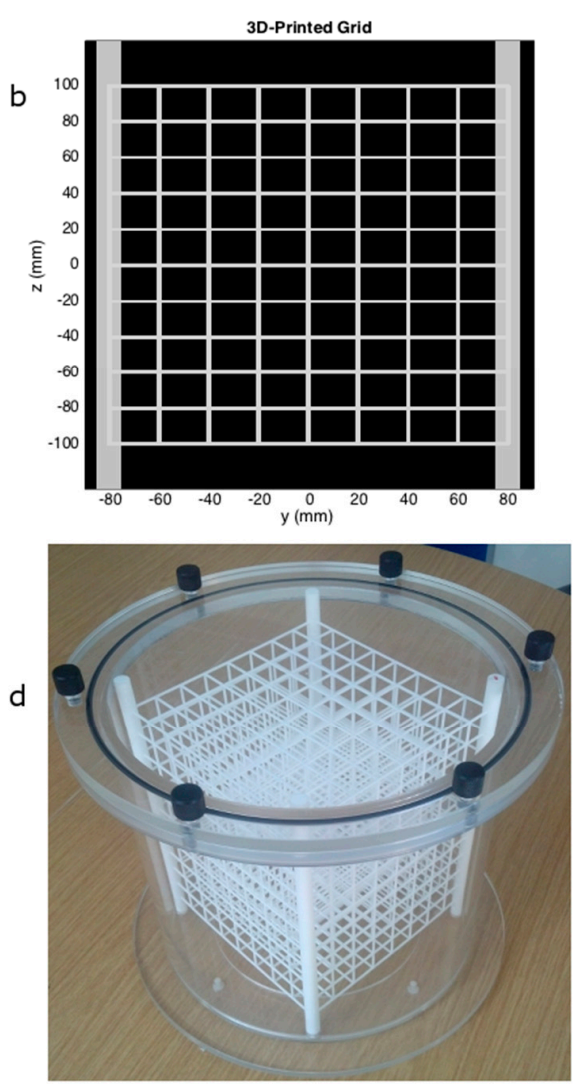

Figure 1. 3D-printed grid in white and strong plastic (a) axial view, (b) sagittal view, (c) perspective view (azimuth $45^{\circ}$, elevation $20^{\circ}$ ), and (d) photograph of the grid fixated inside a leak-tight container. 
Each point of intersection between the mesh lines or edges in 3D space is treated as a reference feature, control point, or vertex. Control point spacing is approximately $20 \mathrm{~mm} \times 20 \mathrm{~mm} \times 20 \mathrm{~mm}$ in the $x, y$, and $z$ dimensions. The volumetric mesh cuboid insert shown in Figure 1 contains a matrix of $9 \times 9$ mesh lines in the $x y$ plane and $9 \times 11$ mesh lines in both $x z$ and $y z$ planes yielding a total of $9 \times 9 \times 11$ (891) control points or vertices. The rest of the phantom was built out of Perspex and assembled in-house in our departmental workshops. The phantom was then filled with off-the-shelf baby oil (Johnson \& Johnson, New Brunswick, NJ, USA). This is a readily available perfumed mineral oil to avoid wavelength-induced artefacts within the imaged structure. The American Association of Physicists in Medicine thus recommends oil over water to reduce such artefacts [3]. The phantom was placed on a laboratory vibrating plated to ensure that air bubbles were not trapped on the 3D-grid as those would cause local susceptibility artefacts. The 3D-printed grid was clearly marked to determine the orientation and the alignment of the phantom in each scan. The cost of printing and raw materials was less than $£ 300$ at time of manufacture (2014).

\subsection{Image Acquisition}

The axis of the cylinder was aligned along the B0 field and supports were used in order to achieve reproducible accurate positioning of the phantom. MR images were acquired using the six MRI scanners listed in Table 1 at both $1.5 \mathrm{~T}$ and $3.0 \mathrm{~T}$ field strengths. For all acquisitions, the body coil and a 3D gradient echo sequence were employed using the acquisition parameters listed in Table 2; the shim volume encompassed the entire phantom and the manufacturers' distortion correction filters were used. A single $k$-space line was encoded per repetition time (TR). The average acquisition time for each scanner was approximately $25 \mathrm{~min}$. The $\mathrm{T}_{1}$ and $\mathrm{T}_{2}$ relaxation values of the baby oil were measured on a 3.0 T Philips Achieva TX system (Philips Healthcare, Best, The Netherlands) using an inversion recovery sequence and a multi-echo sequence, respectively [12]. Mono-exponential fits to both data sets were used to compute relaxation times as $T_{1}=289 \mathrm{~ms}$ and $T_{2}=93 \mathrm{~ms}$.

Table 1. Details of scanners used in this study. Parameters taken from the vendors' manuals (GS = gradient strength, $\mathrm{SR}=$ slew rate).

\begin{tabular}{ccccccccc}
\hline System & Vendor & Model & Field (T) & $\begin{array}{c}\text { GS } \\
(\mathbf{m T} / \mathbf{m})\end{array}$ & $\begin{array}{c}\text { SR } \\
(\mathbf{T} / \mathbf{m} / \mathbf{s})\end{array}$ & $\begin{array}{c}\text { Homogeneity } \\
(\mathbf{p p m})\end{array}$ & $\begin{array}{c}\text { Diameter } \\
(\mathbf{c m})\end{array}$ & $\begin{array}{c}\text { Length } \\
(\mathbf{c m})\end{array}$ \\
\hline A & Philips & $\begin{array}{c}\text { Achieva } \\
\text { TX }\end{array}$ & 3.0 & $\begin{array}{c}40 \\
80\end{array}$ & $\begin{array}{c}200 \\
100\end{array}$ & $\leq 1.8^{1}$ & 60 & 157 \\
\hline B & Siemens & Verio & 3.0 & 45 & 200 & $4.0^{1}$ & 70 & 173 \\
\hline C & Siemens & Avanto & 1.5 & 33 & 125 & $\begin{array}{c}<1.5 \\
(0.8 \text { typical })^{2}\end{array}$ & 60 & 160 \\
\hline D & Siemens & Prisma & 3.0 & 80 & 200 & 1.1 typical ${ }^{2}$ & 60 & 213 \\
\hline E & Siemens & Aera & 1.5 & 45 & 200 & $3.1^{1}$ & 70 & 137 \\
\hline F & Siemens & Aera & 1.5 & 45 & 200 & $3.1^{1}$ & 70 & 137 \\
\hline \multicolumn{1}{l}{ at $50 \times 50 \times 45 \mathrm{~cm}^{3}$ diameter elliptical volume $(\mathrm{DEV}),{ }^{2}$ at $50 \mathrm{~cm}^{3}$ diameter spherical volume (DSV). }
\end{tabular}

Table 2. Imaging parameters for each of the six scanners employed in this study (FA = flip angle, $\mathrm{TR}=$ repetition time; $\mathrm{TE}=$ echo time, $\mathrm{FOV}=$ field-of-view).

\begin{tabular}{cccccccc}
\hline System & Sequence & $\begin{array}{c}\text { TR/TE } \\
(\mathbf{m s} / \mathbf{m s})\end{array}$ & FA $\left.\mathbf{(}^{\circ}\right)$ & $\begin{array}{c}\text { FOV } \\
(\mathbf{m m} \times \mathbf{m m})\end{array}$ & $\begin{array}{c}\text { In-Plane } \\
\text { Resolution } \\
(\mathbf{m m} \times \mathbf{m m})\end{array}$ & $\begin{array}{c}\text { Slice } \\
\text { Width } \\
(\mathbf{m m})\end{array}$ & $\begin{array}{c}\text { Receiver } \\
\text { Bandwidth } \\
\text { (Hz/pixel) }\end{array}$ \\
\hline A & 3D FFE & $11 / 5.2$ & 30 & $361 \times 361$ & $0.71 \times 0.71$ & 1.25 & 136 \\
B & 3D FLASH & $11 / 5.0$ & 10 & $256 \times 256$ & $1.0 \times 1.0$ & 1.0 & 179 \\
C & 3D FLASH & $11 / 5.2$ & 10 & $256 \times 256$ & $1.0 \times 1.0$ & 1.0 & 289 \\
D & 3D FLASH & $11 / 5.0$ & 30 & $361 \times 361$ & $0.71 \times 0.71$ & 1.25 & 285 \\
E & 3D FLASH & $11 / 5.0$ & 30 & $361 \times 361$ & $0.71 \times 0.71$ & 1.25 & 290 \\
F & 3D FLASH & $11 / 5.0$ & 30 & $361 \times 361$ & $0.71 \times 0.71$ & 1.25 & 290 \\
\hline
\end{tabular}


In order to define the true, undistorted control point positions, a corresponding CT scan of the phantom was acquired using a GE LightspeedRT16 system (GE Healthcare, Milwaukee, WI, USA) using an in-plane resolution of $0.71 \mathrm{~mm} \times 0.71 \mathrm{~mm}$ and an out-of-plane resolution of $1.25 \mathrm{~mm}$.

\subsection{Detection of Vertices}

We chose to use normalized cross correlation (NCC) [13] because it is robust and a commonly used similarity measure in image processing. The position of a given pattern (control points) in a three-dimensional image $f$ can be determined by NCC [14]. Let $f(x, y, z)$ denote the intensity value of the image volume $f$ of the size $M_{x} \times M_{y} \times M_{z}$ at the point $(x, y, z)$ where $x \in\left\{0, \ldots, M_{x}-1\right\}$, $y \in\left\{0, \ldots, M_{y}-1\right\}, z \in\left\{0, \ldots, M_{z}-1\right\}$. The pattern is represented by a given template $t$ of the size $N_{x} \times N_{y} \times N_{z}$. Normalized cross correlation value $\gamma$ can be used to find the occurrences of the pattern in image $f$ at each point $(u, v, w)$ for $f$ and the template $t$ which has been shifted by $u$ steps in the $x$ direction, by $v$ steps in the $y$ direction and by $w$ steps in the $z$ direction. The definition for NCC can be extended from $2 \mathrm{D}$ to a third dimension and be expressed as:

$$
\gamma=\frac{\sum_{x, y, z}\left(f(x, y, z)-\bar{f}_{u, v, w}\right)(t(x-u, y-v, z-w)-\bar{t})}{\sqrt{\sum_{x, y, z}\left(f(x, y, z)-f_{u, v, w}\right)^{2} \sum_{x, y, z}(t(x-u, y-v, z-w)-\bar{t})^{2}}}
$$

where $\bar{t}$ is the mean value of the template $t, \bar{f}_{u, v, w}$ denotes the mean value of $f(x, y, z)$ within the area of the template $t$ shifted to $(u, v, w)$ and is calculated by:

$$
\bar{f}_{u, v, w}=\frac{1}{N_{x} N_{y} N_{z}} \sum_{x=u}^{u+N_{x}-1} \sum_{y=v}^{v+N_{y}-1} \sum_{z=w}^{w+N_{z-1}} f(x, y, z)
$$

NCC is a computationally expensive algorithm but it can be approximated using Fourier-based methods or sum-table technique [14,15]. In our application, the reference vertex (Figure 2) is a very well defined feature so an approximation is sufficient to distinguish it from the background as well as the horizontal and vertical edges. We chose an approximation based on Fourier-based methods similar to the implementation given in [16].

The template was determined from the origin of each volume where we could sufficiently capture the central vertex with a template of $7 \times 7 \times 7$ voxels extracted from the iso-centre (origin) of the $\mathrm{CT}$ and MR acquisitions. We assumed that this point in space was free of distortions in the MR acquisition [17] as it is in the magnet iso-centre.

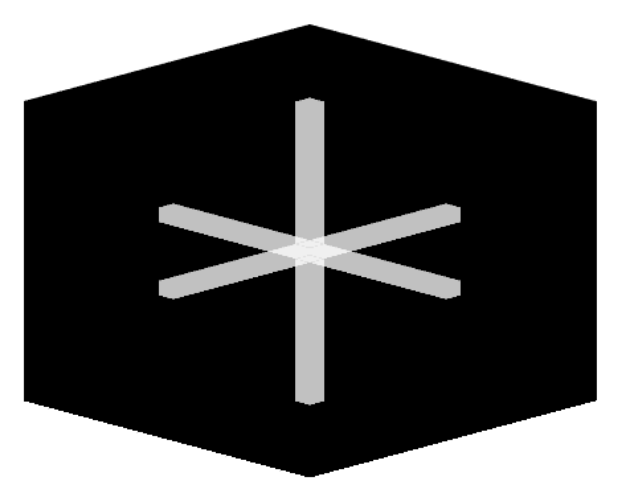

Figure 2. A three-dimensional representation of the reference feature used in normalized cross correlation.

\subsection{Detection of the Spatial Distribution of the Vertices}

The coefficients returned by the NCC algorithm were stored as volume for every voxel traversed in the NCC algorithm. The NCC coefficients were thresholded to leave only the highest correlating 
points corresponding to the vertices. We then determined the vertices using a connected component approach whereby connected image pixels were labelled in groups using a flood-fill algorithm [18]. The spatial coordinates were then determined by finding the centroid of each of the connected pixels by inspecting the statistics of the connected region and subsequently we computed a distance transform of the volumetric lattice with respect to the iso-centre of the volume.

\subsection{Error Metric}

We acquired a CT image of the phantom and calculated the distance transform of the vertices to use as our ground truth $\vec{G}(x, y, z)$ as defined in Equation (3), we chose to use the Euclidean distance metric or L2-norm to measure the geometric distortion, expressed as the distance between the distance transforms of the ground truth volume and the distance transform of the distorted volume $\vec{C}(x, y, z)$ as defined in Equation (4).

$$
\begin{gathered}
\vec{G}(x, y, z)=(x, y, z) \\
d(x, y, z)=\|\vec{G}(x, y, z)-\vec{C}(x, y, z)\|_{2},(x, y, z) \in B^{3}
\end{gathered}
$$

where $d(x, y, z)$ denotes the Euclidean distance and $B^{3}$ denotes the set of distances of the vertices calculated from the iso-centre of the $\mathrm{CT}$ and MR volumes respectively using the distance transform and $\|\cdot\|_{2}$ denotes the L2-norm.

\section{Results}

The ground truth distance map generated from the CT acquisition was compared to an artificially created volume in MATLAB, which matched the control point locations in the 3D-printed insert, and a mean error of $0.0 \mathrm{~mm}$ was found. Measured distances between the control points in the CT dataset were accurate to within $0.05 \mathrm{~mm}$, better than the manufacturer stated value of $0.15 \mathrm{~mm}$. Overall mean error across the entire MR volume for each of the six scanners employed in this study is shown in Table 3. Mean Euclidean distance error for each of the MR volumes was less than $2 \mathrm{~mm}$. Overall, system A produced the best results despite the use of a narrower receiver bandwidth for the scan. Distortion was slightly worse for the $70 \mathrm{~cm}$ diameter bore systems (B, E, F). In Figure 3a,c, 3D surface renderings of the grid phantom for the data acquired using Siemens Prisma 3T (system D) is shown where (azimuth, elevation) in Figure $3 \mathrm{a}, \mathrm{c}$ are $\left(45^{\circ}, 20^{\circ}\right)$ and $\left(225^{\circ}, 20^{\circ}\right)$ respectively. Associated Euclidean distance error volumes are shown in Figure 3b,d respectively. For this particular volume, the maximum Euclidean error was $3.3 \mathrm{~mm}$. To assess the reproducibility of the methodology, the analysis was run several times for a scanner always resulting in identical mean error. Scanning and analysis was also carried out on two occasions for scanner $\mathrm{A}$, leading to near identical distortion maps and identical overall mean error. $(1.1 \pm 0.51 \mathrm{~mm})$.

Table 3. Overall mean error across the entire MR volume for the different MRI scanners.

\begin{tabular}{cc}
\hline System & Mean Error \pm Std $(\mathbf{m m})$ \\
\hline A & $1.1 \pm 0.47$ \\
B & $1.7 \pm 1.3$ \\
C & $1.1 \pm 0.72$ \\
D & $1.1 \pm 0.65$ \\
E & $1.8 \pm 1.1$ \\
F & $1.7 \pm 1.1$ \\
\hline
\end{tabular}



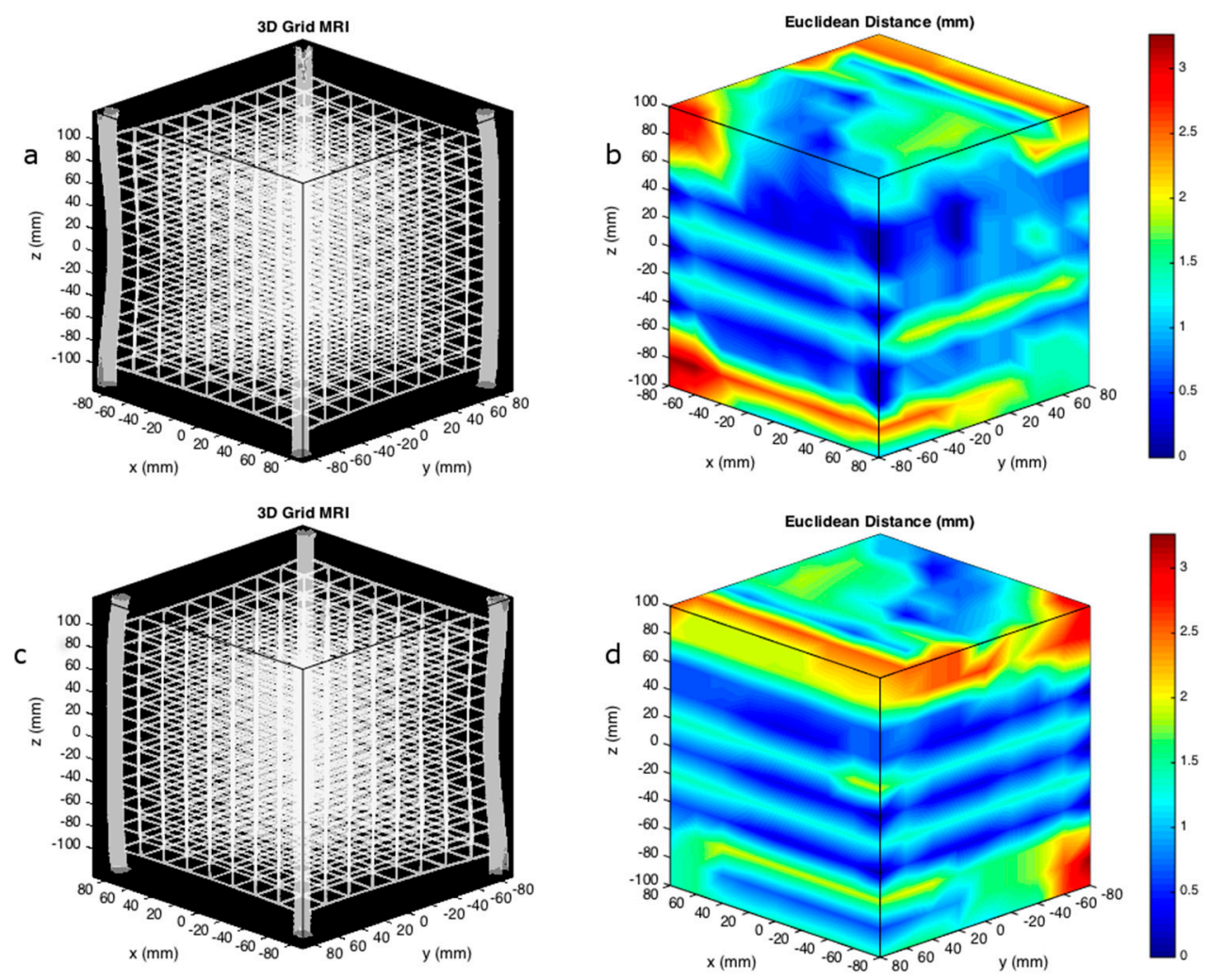

Figure 3. (a) 3D Surface rendering of the grid phantom for the data acquired using system D (azimuth $45^{\circ}$, elevation $20^{\circ}$ ); (b) associated Euclidean distance error volume (azimuth $45^{\circ}$, elevation $20^{\circ}$ ); (c) same as (a) but showing opposing faces (azimuth $225^{\circ}$, elevation $20^{\circ}$ ); and (d) associated error volume (azimuth $225^{\circ}$, elevation $20^{\circ}$ ). Deformation is evident particularly at the edges of the magnetic field.

Deformation in the acquired volume is evident particularly at the edges of the magnetic field. It should also be noted that the deformation in the magnetic field in this particular volume is asymmetric, which led to asymmetry in the computed error volume as reflected in the Euclidean distance error volumes shown in Figure 3b,d. In Figure 4, Euclidean distance error volumes for systems $\mathrm{A}, \mathrm{B}, \mathrm{C}, \mathrm{E}$, and $\mathrm{F}$ are shown where the view in (a) shows (azimuth $45^{\circ}$, elevation $20^{\circ}$ ) and the view in (b) shows (azimuth $225^{\circ}$, elevation $20^{\circ}$ ). Scanners B, E, and F resulted in the largest Euclidean distance error with maximum detected errors of $6.2,6.9$, and $6.0 \mathrm{~mm}$ respectively. The maximum detected error in scanners $A$ and $C$ were $2.4 \mathrm{~mm}$ and $2.7 \mathrm{~mm}$ respectively. These results correlate well with the manufacturer stated homogeneity presented in Table 1.

Mean Euclidean distance errors for the axial and sagittal control point planes for all scanners are given in Figure 5a,b respectively. Distortion in the central planes for all scanners was less than $2 \mathrm{~mm}$. Some oscillations in the distortion error are visible on all systems, especially in the central part (Figure 5a) along the $\mathrm{z}$ axis. Those are likely to be the results of remaining unwanted field harmonics [19] not fully corrected by shimming over the entire object. 

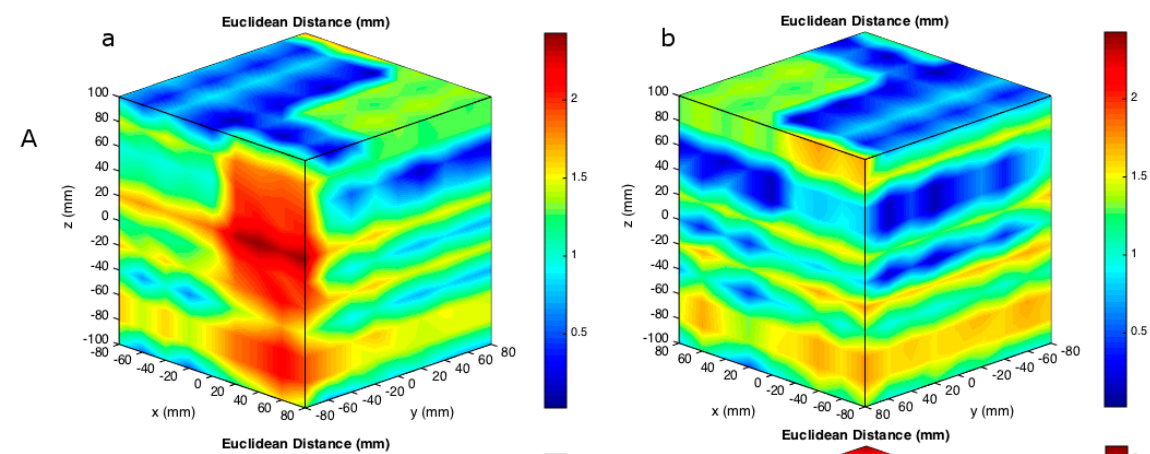

B
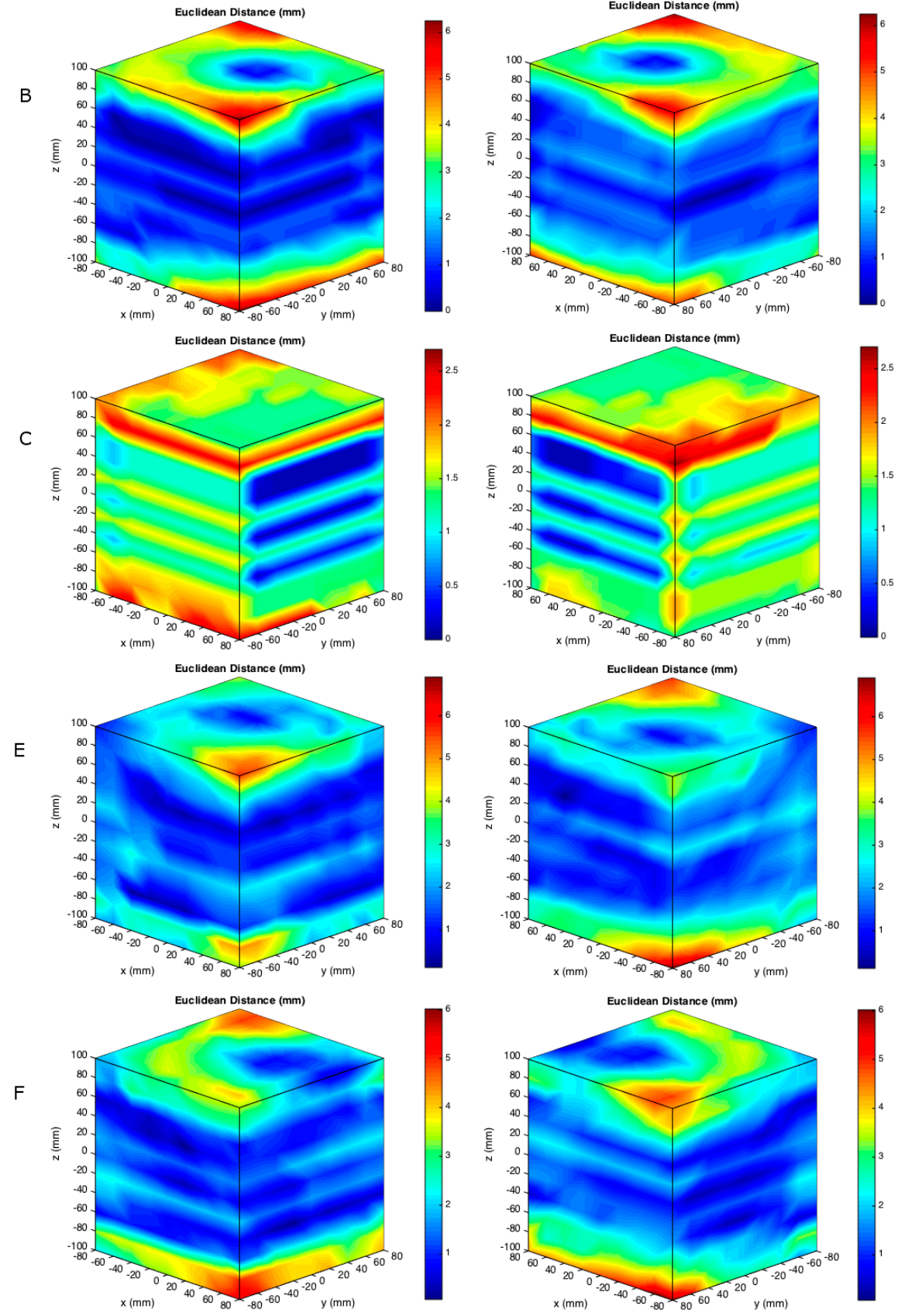

Figure 4. Euclidean distance error volumes for systems A, B, C, E and F (a) (azimuth $45^{\circ}$, elevation $20^{\circ}$ ) and (b) (azimuth $225^{\circ}$, elevation $20^{\circ}$ ). For system D see Figure 3. 

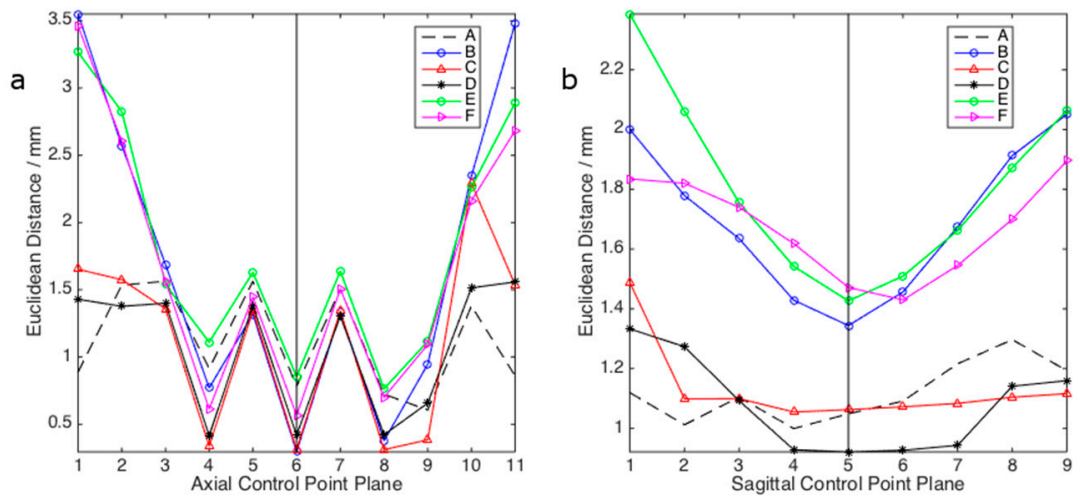

Figure 5. Mean Euclidean distance in $\mathrm{mm}$ for the axial (a) and sagittal (b) control point planes of the phantom. The vertical line in each figure represents the central control point plane in both orientations.

\section{Discussion}

In this paper, we explored the detection of reference features of a 3D printed phantom to assess MRI distortion in six clinical scanners from two vendors. These reference features were "point-like" objects or vertices, which resulted from the intersection of mesh lines in 3D space. We chose a custom design such that the distance between these reference features in $x, y$ and $z$ directions was $20 \mathrm{~mm}$. Although distortions of up to $25 \mathrm{~mm}$ at $1.5 \mathrm{~T}$ field strength have been reported [17], mean Euclidean distance error for all MR volumes in this study was less than $2 \mathrm{~mm}$. The conclusions in this study agree well with many other studies that indicated that MRI is quite accurate near the centre of the field but is spatially inaccurate toward the edges of the magnetic field [20]. Detecting spatial accuracy of coordinates in 3D space is essential for determining geometric distortion to aid in MR/CT planning and for general quality assurance in MRI. In this study, the manufacturers' distortion filters were used to replicate our institution clinical practice; however, the phantom could also be used to assess filter efficacy by acquiring data with or without them.

The accuracy of volume measurements is an important topic in MRI, as volumetry can represent an essential tool in neurology [21] or oncology [22]. As the phantom used in this study is comprised of $20 \times 20 \times 20 \mathrm{~mm}^{3}$ cubes defined by the plastic mesh, it could be used to check volume accuracy. However, a finer mesh might be recommendable depending on the size of the volumes studied, for example for prostate volumetry.

Accurate positioning of the phantom is important to ensure that the axes of the phantoms are aligned with the axes of the scanner; this avoids misalignment between the MRI scans and the reference. Although physical supports were used to achieve the alignment, it was felt that the design could have been improved to easily clip the phantom onto the supports or a cradle. Design improvements could also be made in the 3D-printed insert whereby a larger feature such as sphere can represent a control point. This will then alleviate the need for manual inspection of the 3D normalized cross correlation, as geometric distortions will often make thresholding of the NCC coefficients difficult. Image erosion may then be a viable technique for the detection of the vertices. One could also choose to minimize the gap between these reference features further for example reducing the gap to $5.0 \mathrm{~mm}$ or alternatively increase the dimensions of the volume in order to increase the number of control points contained within the volume. A larger spatial volume will assist in having a better understanding of the magnetic field distortion. This depends on the success of having an alternative design printed in 3D, which in turn depends on the employed support material. Nevertheless, this is a simple and cost-effective approach to determine the spatial accuracy of control points within an imaged volume.

Author Contributions: The manuscript was prepared by Maysam Jafar and discussed and corrected with Marc E. Miquel. The 3D printed phantom was designed by Maysam Jafar with input from Marc E. Miquel. MRI data 
was acquired by Maysam Jafar and CT data by Chris Dean. Analysis code was developed by Maysam Jafar and Yassir M. Jafar.

Conflicts of Interest: The authors declare no conflict of interest.

\section{References}

1. Sumanaweera, T.S.; Adler, J.R.; Napel, S.; Glover, G.H. Characterization of spatial distortion in magnetic resonance imaging and its implications for stereotaxic surgery. Neurosurgery 1994, 35, 696-703. [CrossRef] [PubMed]

2. Wang, D.; Doddrell, D.M.; Cowin, G. A novel phantom and method for comprehensive 3-dimensional measurement and correction of geometric distortion in magnetic resonance imaging. Magn. Reson. Imaging 2004, 22, 529-542. [CrossRef] [PubMed]

3. Price, R.R.; Axel, L.; Morgan, T.; Newman, R.; Perman, W.; Schneiders, N.; Selikson, M.; Wood, M.; Thomas, S.R. Quality assurance methods and phantoms for magnetic resonance imaging-Report of AAPM nuclear magnetic resonance task group no-1. Med. Phys. 1990, 17, 287-295. [CrossRef] [PubMed]

4. Kawanaka, A.; Takagi, M. Estimation of static magnetic field and gradient fields from NMR image. J. Phys. E Sci. Instrum. 1986, 19, 871-875. [CrossRef]

5. Mizowaki, T.; Nagata, Y.; Okajima, K.; Kokubo, M.; Negoro, Y.; Araki, N.; Hiraoka, M. Reproducibility of geometric distortion in magnetic resonance imaging based on phantom studies. Radiother. Oncol. 2000, 57, 237-242. [CrossRef]

6. Walton, L.; Hampshire, A.; Forster, D.M.C.; Kemeny, A.A. A phantom study to assess the accuracy of stereotactic localization, using T1-weighted magnetic resonance imaging with the Leksell stereotactic system. Neurosurgery 1996, 38, 170-176. [CrossRef] [PubMed]

7. Walton, L.; Hampshire, A.; Forster, D.M.C.; Kemeny, A.A. Stereotactic localization with magnetic resonance imaging: A phantom study to compare the accuracy obtained using two-dimensional and three-dimensional data acquisitions. Neurosurgery 1997, 41, 131-137. [CrossRef] [PubMed]

8. Yu, C.; Apuzzo, M.L.J.; Zee, C.S.; Petrovich, Z. A phantom study of the geometric accuracy of computed tomographic and magnetic resonance imaging stereotactic localization with the Leksell stereotactic system. Neurosurgery 2001, 48, 1092-1098. [CrossRef] [PubMed]

9. Sumanaweera, T.; Glover, G.; Song, S.; Adler, T.; Napel, S. Quantifying MRI geometric distortion in tissue. Magn. Reson. Med. 1994, 31, 40-47. [CrossRef] [PubMed]

10. Baldwin, L.N.; Wachowicz, K.; Thomas, S.D.; Rivest, R.; Fallone, B.G. Characterization, prediction, and correction of geometric distortion in 3 T MR images. Med. Phys. 2007, 34, 388-399. [CrossRef] [PubMed]

11. Breeuwer, M.; Holden, M.; Zylka, W. Detection and correction of geometric distortion in 3D MR images. Proc. SPIE 2001, 4322. [CrossRef]

12. Tofts, P.S.; Duboulay, E.P.G.H. Towards quantitative measurements of relaxation-times and other parameters in the brain. Neuroradiology 1990, 32, 407-415. [CrossRef] [PubMed]

13. Gonzalez, R.C.; Woods, R.E. Digital Image Processing, 2nd ed.; Prentice Hall: Upper Saddle River, NJ, USA, 2001.

14. Briechle, K.; Hanebeck, U. Template Matching using Fast Normalized Cross Correlation. Proc. SPIE 2011, 4387. [CrossRef]

15. Crow, F.C. Summed-area Tables for Texture Mapping. SIGGRAPH Comput. Graph. 1984, 18, $207-212$. [CrossRef]

16. Kroon, D.-J. Fast/Robust Template Matching. Matlab File Exchange, 2009. Available online: https:/ / uk.mathworks.com/matlabcentral/fileexchange/24925-fast-robust-template-matching? requestedDomain=www.mathworks.com (accessed on 28 April 2017).

17. Doran, S.J.; Charles-Edwards, L.; Reinsberg, S.A.; Leach, M.O. A complete distortion correction for MR images: I. Gradient warp correction. Phys. Med. Biol. 2005, 50, 1343-1361. [CrossRef] [PubMed]

18. Vincent, L. Morphological grayscale reconstruction in image analysis: Applications and efficient algorithms. IEEE Trans. Image Process. 1992, 2, 176-201. [CrossRef] [PubMed]

19. Hillenbrand, D.F.; Lo, K.M.; Punchard, W.F.B.; Reese, T.G.; Starewicz, P.M. High-order MR shimming: A simulation study of the effectiveness of competing methods, using an established susceptibility model of the human head. Appl. Magn. Reson. 2005, 29, 39-64. [CrossRef] 
20. Orth, R.C.; Sinha, P.; Madsen, E.L.; Frank, G.; Korosec, F.R.; Mackie, T.R.; Mehta, M.P. Development of a unique phantom to assess the geometric accuracy of magnetic resonance imaging for stereotactic localization. Neurosurgery 1999, 45, 1423-1429. [CrossRef] [PubMed]

21. Keller, S.S.; Roberts, N. Measurement of brain volume using MRI: Software, techniques, choices and prerequisites. J. Anthropol. Sci. 2009, 87, 127-151. [PubMed]

22. Harvey, H.; Orton, M.R.; Morgan, V.A.; Parker, C.; Dearnaley, D.; Fisher, C.; deSouza, N.M. Volumetry of the dominant intraprostatic tumour lesion: intersequence and interobserver differences on multiparametric MRI. Br. J. Radiol. 2017, 90, 20160416. [CrossRef] [PubMed]

(C) 2017 by the authors. Licensee MDPI, Basel, Switzerland. This article is an open access article distributed under the terms and conditions of the Creative Commons Attribution (CC BY) license (http:/ / creativecommons.org/licenses/by/4.0/). 